\title{
Gamification and Its Applications
}

\author{
KDV PRASAD \\ Institute of Industrial \& Human Resources Development (IIHRD), \\ Financial city, Gachibowli, Hyderabad, India.
}

\begin{abstract}
In the recent past, gamification is a new buzzing word and is used frequently by researchers in particular in the area of management. Gamification is applying gaming and using gaming components and procedures in a nongaming environment. The three main components of gamification are game elements - a design prototype which can be integrated into the game; game design - A complete procedure building engaging games, based on business objectives, technical feasibility and management expectations; the last one is the non-game environment - The non-gaming environment is the components involved in real-world business and social impact objectives and goals. If gamification is applied correctly in a business or social environment, the results will be amazing and can inspire the business community decisions. Gamification can be the future of the business and can make the business environment the gaming components fun and those fun and appealing components can be applied in real-life business activities. To make gamification effective the game dynamics, game design, behavioural change, and motivation are the aspects that need to be combined applied in non-gaming environment. This brief note presents the main ideologies that can the gamification design in the business environment more successful. The author also narrates whene the gamification procedures can be useful in the business world.
\end{abstract}

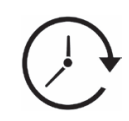

Article History

Received: 23 September 2021

\section{Keywords}

Environment; Element; Gamification; Game Design; Procedure;

\section{Introduction}

The idiom 'gamification' was devised by Nick Pelling in 2002, however, the businesses accepted this word only in the recent past. In the business industry, the gamification technique uses badges, points and leader boards to enhance productivity and employee engagements. The awards and badges motivate the employees and enhance productivity and created an improved workplace environment (Prasad et al., 2019). In the recent past, several business houses

CONTACT KDV Prasad 1 k.d.prasad@cgiar.org 9 Institute of Industrial \& Human Resources Development (IIHRD), Financial city, Gachibowli, Hyderabad, India.

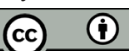

(C) 2021 The Author(s). Published by Enviro Research Publishers.

This is an Open Access article licensed under a Creative Commons license: Attribution 4.0 International (CC-BY).

Doi: http://dx.doi.org/10.12944/JBSFM.03.01-02.02 
Vol. 03(1-2) 04-07 (2021)

are incorporating gamification mechanisms with rewards to enhance employee motivation (Gartner

The three components the gamification mechanism uses are badges, points and leader boards. Several studies reported the employees who received or were awarded badges based on their performance responded proactively and in turn improved at the workplace environment and increased performance. Gartner (2015) reported that several organizations embracing gaming mechanisms and rewards to motivate employees and customers. Gamification is a prospective strategy to achieve business development goals. The gamification strategies are not limited to business houses, but also be implemented in education, health sector, human resources, recruitment, learning and development, talent management and research institutes. It was evident that gamification is more productive where human engagement is in decline in the business houses and educational settings Fitz-Walter (2011). Saima Hussain et al. (2018) applied gamification effect on employee motivation, retention and engagement loyalty and employee commitment to the organization using 142 University students. This study divided the participants into two groups the gamified and non-gamified groups. The study concluded the group under gamified environment showed increased motivation, engagement, retention and less turnover.

Aman Jain and Debolina Dutta (2018) reported the use of Guerilla tactics making learning fun in the millennial cohort. The authors concluded that gamification can help effectively design and integrate new learning procedures using gamified learning modules in general and Millennial Cohort in particular. Incorporating fun and playfulness in the workplace and introducing rewards based on performance make the employee more loyal to the organization (Basten, 2017). The basic essence of gamification in a business environment is the inclusion of fun, games, fun playful than a serious stern working environment (Werbach and Hunder, 2012). Gamification is still new in some areas, and most of the present approaches are mainly focussing on game elements, which is not an appropriate approach as most of the gamification components are about issues related to psychology (Zicherman and Cunningham 2011). The behaviour of an individual and the culture also influences gamification. The psychological concepts are integrated with the gamification techniques to motivate the customers, employees, and human beings irrespective of the area. The gamification enhances both the intrinsic and extrinsic motivation and behavioural change of a human being. Gamification is widely used for behavioural change including individual, cultural and social environments ( AlMarshedi et al., 2017).

\section{Gamify, What?}

The users of the gamification techniques must decide what or in which area they want to apply the gamification techniques. There are several areas where gamification can be applied successfully with good game design techniques integrating playfulness and fun in the respective area. Enhancing employee engagement, enhance employee performance, recruitment, learning and development to enhance student engagement, enhancing the engagement of Academicians of higher education, functions of human resources, reducing the absenteeism making assignments more exiting integrating fun playful elements in the assignments are some of the areas can be gamified successfully for saving the resources, employee cost and enhance productivity.

\section{Application of Gamification}

The gamification is now being applied in several industries business, health care, education, military, research, BPO, information technology but not limited to. The following section presents the evidence-based application of gamification in the areas as described.

\section{Employee Engagement}

Several studies reported that the application of gamification techniques enhance employee engagement, motivation and performance. Prasad et al. (2019) in a case study with an eCommerce company successfully combined gamification and resource pooling methodologies to enhance operational efficiency and productivity and saving resources. The eCommerce industry is a market with diverse individuals with multicultural customers. Therefore, the pooling of resources was successfully done with a perfect blend of gamification to motive stakeholders and enhance business opportunities. In another study, authors applied stimulation of games, behavioural change and intrinsic and extrinsic motivation as the areas of gamification to 
Vol. 03(1-2) 04-07 (2021)

improve the work quality, to remove the mundane and expensive structures, to automate routine activities. The authors adapted a survey method to differentiate gaming and non-gaming environments. The multiple regression analysis revealed enhanced employee engagement and improvement of quality of work and a decrease in turnover absenteeism (Prasad and Mruthyanjaya Rao, 2021). Prasad et al. (2020) reported enhanced engagement of higher education faculty and teaching staff using the gamification interventions. The authors carried out a General Linear Model analysis to dissect the results on pre and post-gamification interventions for motivation, engagement dedication and loyalty to the organization using a questionnaire. The results indicated that gamification influenced of the factors studied are statistically significant and a good predictor of turnover and loyalty. The teaching staff engagement is enhanced using applying the gamification techniques by motivation.

\section{Learning and Development}

The gamification methods can be integrated to engage students, build independence and enhance student academic success. Carla Wilson (2015) in a study on enhancing student engagement with gamification created a gamified learning environment that consists of a series of quests with a single narrative. The study was implemented using the practical application of education strategies. The peers reported positive feedback as the student engagement was enhanced and gamification can be a serious strategy in future in the learning and development area. The gamification environment used in this study is online with face to face environment. The fun, playful, and entertaining in gamification helps nurture engagement and motivate the learner to be more amenable to interactions with enhanced learning content. The gamified learning environment motivates to engage. However, sound learning principles and playful actions need to be integrated to enhance in learning interest. Quests, mystery plots, game labs, are used to motivate the students. The gamification can be a potential tool for enhanced student engagement by framing an activity as a game in learning to enhance motivation (FitzWalter, 2013). Deterding et al. 2010 suggested the use of structural designs, incorporating game-based practices with game components and embedding the characteristics of games in a non-game environment for student motivation and enhanced learning.

\section{Gamification for Health Promotion}

Edwards et al (2016) used carried out a systematic review of health applications with gaming components analyzing embedded behavior change methods. The applications developed embedding behaviour change techniques following the standard taxonomy protocols. The health application which is embedded with gamification use a variety of behavior change techniques that are installed in the smartphone. The authors observe there is a weak correlation between game content and behaviour change. The authors suggest there should a strong collaboration between behavior scientists, health professionals, and application developers to make the smartphone apps for behavior change more effective. There is a need to develop standard protocols to evaluate the apps with experimental trials.

\section{Human Resources}

The gamification can be effectively used in the rea of recruitment making recruitment strategy fun, playful and amazing. By adding game elements into the recruitment strategy the candidate can be immersed seriously in recruiting process so most of his/her talent can be assessed. The gamification in recruitment can relieve old and mundane methods of recruitment protocols as the candidate attending the interview will think more in a gaming environment. Google Code Jam, where the programmers rest their programming logic skills to solve algorithmic puzzles, using $\mathrm{C}++$, JavaScript, etc. This is fun and challenging for to individuals enhance their programming skills and attract the right talent. Domino's Pizza Moghul is another example where the customers can design their pizzas and earn rewards. If suitable some customers can be potential job applicants. This entire application builds on innovation, fun, creativity and novel ideas.

\section{Conclusions}

For any successful gamification methodology, the game design needs to be perfect for effective implementation. The implementer should think and assess strategically whether the gamification and game design is suitable for applying the choice of his/ her area. While designing the games care should be taken that games will have engaging experiences and motivates desired behaviors. The gaming elements points, badges, leader boards should be planned appropriately as these provide important feedback to the game designer. Badges will have a positive 
Vol. 03(1-2) 04-07 (2021)

effect on motivation. Leaderboards indicate where a person stands relative to his/her peer. All these gaming elements need to be efficiently designed before applying gamification. The Management will/interest to apply the gamification also plays an important role in effectively implementing the gamification. One can not rush things in gamification for faster results.

\section{Funding}

The author(s) received no financial support for the research, authorship, and/or publication of this article.

\section{Conflict of Interest}

The authors do not have any conflict of interest

\section{References}

1. AlMarshedi, A., Wanick, V., Wills, G. B., \&Ranchhod, A. (2017). Gamification and behaviour. In Gamification (pp. 19-29). Springer, Cham.

2. Basten, D. (2017). Gamification. IEEE Software, 34(05), 76-81.

3. Deterding, S., Dixon, D., Khaled, R., \&Nacke, L. (2011, September). From game design elements to gamefulness: defining" gamification". In Proceedings of the $15^{\text {th }}$ international academic MindTrek conference: Envisioning future media environments (pp. 9-15).

4. Edwards, E. A., Lumsden, J., Rivas, C., Steed, L., Edwards, L. A., Thiyagarajan, A., ... \& Walton, R. T. (2016). Gamification for health promotion: systematic review of behaviour change techniques in smartphone apps. BMJ open, 6(10), e012447.

5. Fitz-Walter, Z., Tjondronegoro, D., \& Wyeth, P. (2011, November). Orientation passport: using gamification to engage university students. In Proceedings of the 23rd Australian computerhuman interaction conference (pp. 122-125).

6. Gartner Research. 'Gartner says by 2015 , more than 50 per cent of organisations that manage innovation process will gamify those processes,' Press release 12 April 2011. www,.gartner.com/newsroom/id/162914 (accessed 7 February 2014)

7. Hussain, S., Qazi, S., Ahmed, R. R., Streimikiene, D., \&Vveinhardt, J. (2018). Employees management: evidence from gamification techniques. Montenegrin Journal of Economics, 14(4), 97-107.

8. Jain, A., \& Dutta, D. (2019). Millennials and gamification: guerilla tactics for making learning fun. South Asian Journal of Human Resources Management, 6(1), 29-44.
9. Lawanda, N., Mohile, R., \&SagarikaDatta, S. (2018). Gamification and Employee Engagement: Theoretical Review on the Role of HR. In International Conference on Digital Innovation: Meeting the Business Challenges. Pp 183-198.

10. Pelling, N. The (short) prehistory of "gamification"... Funding Startups (\& other impossibilities). 2011. Available at: https:// nanodome.wordpress.com/2011/08/09/theshortprehistory-of-gamification/ [Accessed September 16, 2015]

11. Prasad, K. D. V., \& Mangipudi, M. R. (2020). Enhanced Academicians Engagement: Evidence From Gamification Interventions In Higher Education Institutes. International Journal Of Engineering Technologies And Management Research, 7(9), 24-30.

12. Prasad, K.DV, \&Mruthyanjaya Rao, M. (2021). Gamification for Employee Engagement: An Empirical Study With E-Commerce Industry. Montenegrin Journal of Economics, 17(2), 145-156.

13. Prasad, K.D.V., Mruthyanjaya Rao M \& Rajesh Vaidya, Gamification and Resource Pooling for Improving Operational Efficiency and Effective Management of Human Resources: A Case Study with an Ecommerce Company, International Journal of Management (IJM), 10 (6), 2019, pp. 76-87.

14. Werbach, K., \& Hunter, D. (2012). For the win: How game thinking can revolutionize your business. Wharton digital press.

15. Wilson, C. M. (2015). Increasing student engagement through gamification.

16. Zichermann, G., \& Cunningham, C. (2011). Gamification by design: Implementing game mechanics in web and mobile apps. "O'Reilly Media, Inc.". 